\title{
Proactive interference from items previously stored in visual working memory
}

\author{
Tal Makovski and Yuhong V. Jiang \\ Harvard University, Cambridge, Massachusetts \\ and University of Minnesota, Minneapolis, Minnesota
}

\begin{abstract}
This study investigates the fate of information that was previously stored in visual working memory but that is no longer needed. Previous research has found inconsistent results, with some showing effective release of irrelevant information and others showing proactive interference. Using change detection tasks of colors or shapes, we show that participants tend to falsely classify a changed item as "no change" if it matches one of the memory items on the preceding trial. The interference is spatially specific: Memory for the preceding trial interferes more if it matches the feature value and the location of a test item than if it does not. Interference results from retaining information in visual working memory, since it is absent when items on the preceding trials are passively viewed, or are attended but not memorized. We conclude that people cannot fully eliminate unwanted visual information from current working memory tasks.
\end{abstract}

What is the fate of information stored in visual working memory (VWM) after it is no longer needed? Given the limited capacity of VWM (Luck \& Vogel, 1997), it may be advantageous to erase unwanted information to make room for new memory. The idea that unwanted information can be effectively released from VWM receives some support from recent studies; for example, attentional capture by a singleton distractor is increased if that distractor matches features currently held in VWM but not if it matches features previously held in VWM(Olivers, Meijer, $\&$ Theeuwes, 2006). These results suggest that the contents of VWM can be flexibly updated, depending on the current behavioral goal. The idea that only current information is held in VWM is also implicitly assumed in standard measures of VWM capacity, which is usually measured by plotting performance as a function of the current trial's memory load (Cowan, 2001; Pashler, 1988) without considering lingering memory load from preceding trials.

However, research on source memory has shown that humans are imperfect at discarding previous memories and that previous memories often intrude into current memory (Jacoby, Kelley, Brown, \& Jasechko, 1989; Johnson, 1997). Interference from previous trials' memories on current memory tasks is known as proactive interference (Crowder, 1976; Keppel \& Underwood, 1962). It is typically explained as lingering familiarity of previously remembered information that is later confused with the current trial's memory. Studies on proactive interference, however, have focused primarily on memory for verbal materials or memory for nameable, pictorial materials (Jonides \& Nee, 2006). Given that VWM for unnameable visual materials is highly limited (Luck \& Vogel, 1997), and that this memory quickly dissi- pates upon presentation of new visual inputs (Landman, Spekreijse, \& Lamme, 2003; Makovski \& Jiang, 2007), it is possible that performance on a current VWM task is resistant to proactive interference (Olivers et al., 2006).

To date, studies on VWM have restricted their scope to investigating memory acquired from isolated trials, even though each VWM trial is embedded within the context of other VWM trials. The present study is our first attempt to characterize intertrial effects in VWM. The main goal of this study is to investigate interference from previously processed items on current VWM performance. We ask the following questions. First, can recently encountered visual information interfere with current VWM performance? If so, is proactive interference modulated by whether the interfering stimulus was actively remembered or was passively viewed? Second, is visual proactive interference spatially specific, with greater interference from previous items that spatially overlap with the current memory? If so, what is the spatial reference frame for the spatial specificity?

To test proactive interference in VWM, we modified the recent-probes paradigm (Monsell, 1978) and combined it with a modified change detection task (Rensink, 2002). In our task, participants are first shown several visual objects to remember. After a short retention interval, a test stimulus is presented for participants to judge whether it matches their memory. The test item can either match or mismatch one of the memory items. Critically, we manipulated the type of mismatches so that the mismatch of the current trial sometimes matched visual input from the previous trial. If proactive interference occurred, participants would be more likely to commit false alarms when the mismatch was the same as the previous trial's input than when the 
mismatch was a new item not presented in the previous trial. Additionally, by manipulating the nature of processing required on the previous trial's input (active memory, attending without memorizing, or passive viewing), and by varying the spatial relationship between the test probe and trial $n-1$ 's items, we could address questions related to visual proactive interference and its spatial specificity.

\section{EXPERIMENT 1 \\ Visual Proactive Interference in a Color VWM Task}

Can visual input seen on trial $n-1$ interfere with VWM performance on trial $n$ ? There are several possibilities, depending on how much control humans have over the content of VWM. The flexible control hypothesis predicts that we are capable of controlling the content of VWM that only currently relevant information is held there. In turn, information from previous trials is effectively released from VWM. That information may enter other kinds of memory, such as visual long-term memory, but it will not intrude into VWM on trial $n$. Consequently, very little proactive interference is expected from previously encountered items that are no longer needed in a current VWM task. This hypothesis is consistent with Olivers et al.'s (2006) findings. Alternatively, the limited control hypothesis predicts that we have limited control over the access of information to VWM, allowing previously encountered information to intrude into current VWM. The intrusion may occur as a consequence of not releasing previously memorized items from VWM, or it may occur because that information is released but then brought back into VWM from other memory stores. In either case, the intrusion is expected to lead to proactive interference on trial $n$ 's VWM performance.

To evaluate these competing hypotheses, we tested VWM for an array of colors on trial $n$. Observers were also required to remember colors on trial $n-1$. We then measured the degree of intrusion in VWM performance from objects encountered on trial $n-1$ and from new objects. This experiment also allowed us to test spatial specificity of this intrusion.

\section{Method}

Participants. Participants tested in all experiments were volunteers from Harvard University and community. They signed an informed consent before the experiment and were compensated for their time. All had normal color vision and normal or corrected-tonormal visual acuity. There were 16 participants in Experiment 1; mean age, 22.7 years.

Equipment. Participants were tested individually in a normally lit interior room. They sat, unrestricted, about $57 \mathrm{~cm}$ from a computer monitor. The experiments were programmed with Psychophysics Toolbox (Brainard, 1997; Pelli, 1997) implemented in MATLAB.

Stimuli and Task. On each trial a memory array of five colored disks (diameter $=1.31^{\circ}$ ) was presented for $1,000 \mathrm{msec}$ and erased. The items were presented equidistantly on an imaginary circle; the center of each item was $4.51^{\circ}$ from fixation. After a blank retention interval of 1,000 msec, a single test color was presented at one of the five memorized locations. The test item was erased upon a keypress response. Participants were asked to report, by pressing one of the two keys, whether or not the test color matched the memory color at that location. The test item matched the color of the memory item at that location on $40 \%$ of trials, and mismatched on the remaining $60 \%$ of trials. The mismatch trials could be either a novel baseline (20\%), where the test item was a novel color not presented on the current trial or on trial $n-1$, or a previous match (40\%), in which the test item was different from the current trial's memory items but was the same as one of the unprobed colors on trial $n-1$. There were two types of previous match trials: same-location trials, in which the probe matched the trial $n-1$ item in both location and color $(20 \%$ of trials); and different-location trials, in which the probe matched the trial $n-1$ item in color but not in location (20\% of trials). Figure 1 illustrates the four types of probes: (1) match; (2) mismatch, novelbaseline; (3) previous-match, same-location; and (4) previous-match, different-location.

Procedure. Each participant completed 250 experimental trials divided into 5 blocks. The first trial in each block was always a match trial. Participants were told to respond as accurately as possible; speed was not emphasized. After each incorrect response, they received accuracy feedback immediately, in the form of an error beep. The next trial started $500 \mathrm{msec}$ after the response. To minimize verbal recoding, participants were required to rehearse a three-letter word as quickly as they could throughout the block. The rehearsed word was specified at the beginning of each block.

\section{Results}

Accuracy rates for different types of probe trials are plotted in Figure 2. Statistical analyses were conducted on accuracy of the mismatch trials, since match trials were uninformative about proactive interference. ${ }^{1}$

Participants made more intrusion errors on previousmatch trials than on novel-baseline trials, but only when the probe matched the previous trial's memory in both color and location (previous-match, same-location) $[t(15)=$ $4.49, p<.01]$. When the probe matched the previous trial's memory in color but not in location, performance was indistinguishable from that of the novel-baseline $[t(15)=$ $1.23, p>.20]$, and was significantly better than that in the previous-match, same-location condition $[t(15)=2.31$, $p<.05]$. Thus, when items on trial $n-1$ were committed to VWM, they resulted in significant proactive interference, but interference was spatially specific.

Although speed was not emphasized, we also analyzed reaction time (RT) data to ensure that there was no speedaccuracy trade-off. Mean RTs for correct trials ranged from 840 to $880 \mathrm{msec}$ in the mismatch conditions. This difference was not significant $[F(2,30)=1.54, p>.20]$. Similarly, Experiments 2 and 3 reported below also revealed no evidence for speed-accuracy trade-off. We will only report accuracy data in these experiments.

\section{Discussion}

Experiment 1 clearly showed that the degree of control humans have over the content of their VWM is somewhat limited; information no longer needed can linger in memory and interfere with current VWM performance. This experiment also revealed spatial specificity of proactive interference in VWM. Proactive interference from previously memorized items is significantly reduced when the probe item matches the color but not the location of that memory. Thus, the memory that is not fully released from a previous trial is not entirely abstract; it retains specific visual properties, such as the location of the previous memory. In addition to spatial locations, other irrelevant 


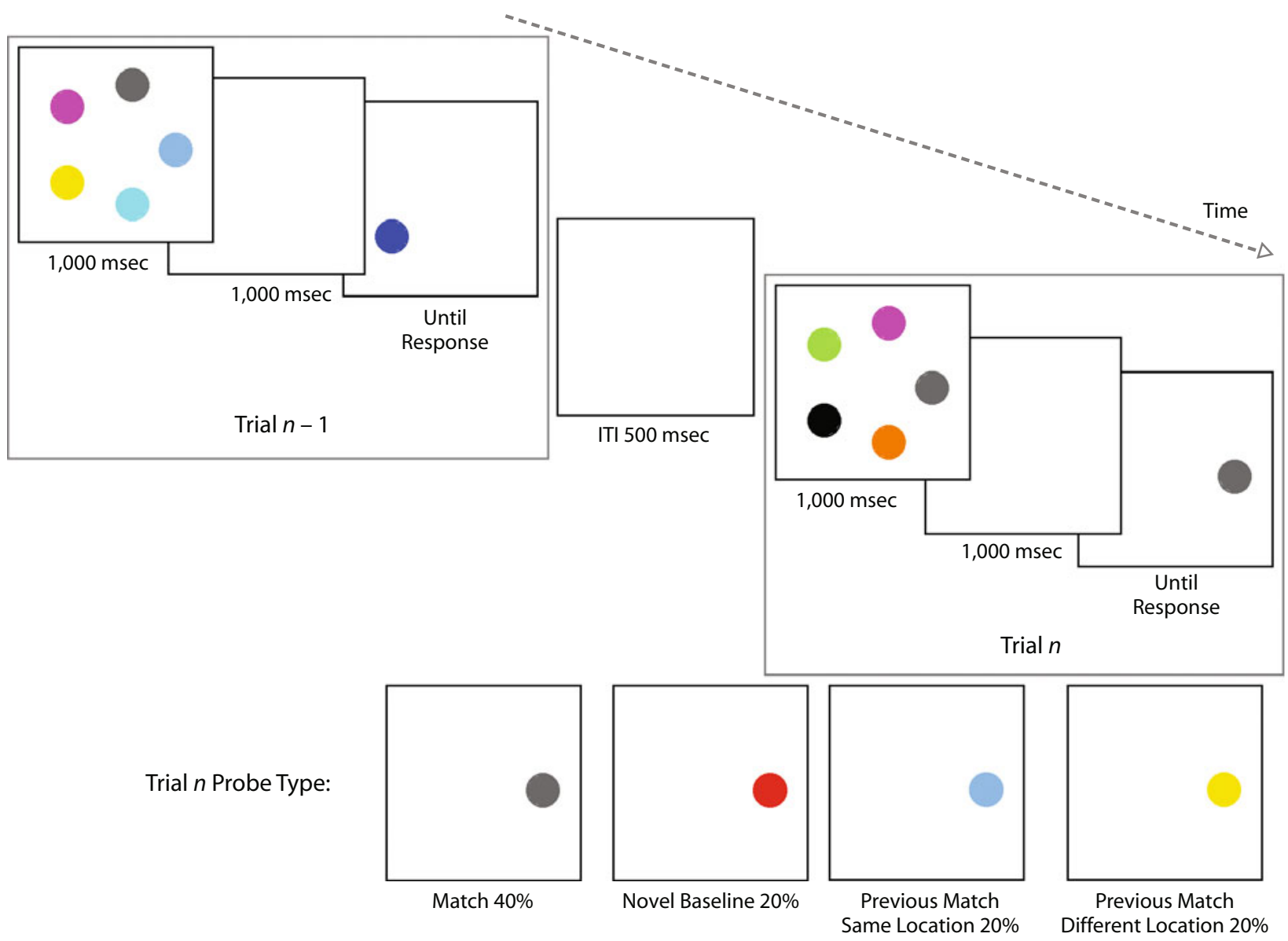

Figure 1. A schematic illustration of trial sequences used in Experiment 1. The four types of probes (for trial $n$ ) are shown at the bottom.

feature dimensions, such as item shape in a color VWM task, may also modulate the size of proactive interference. The strength of the modulation may vary, depending on the saliency of the irrelevant feature dimension.

Experiment 1 is important in establishing a proactive interference effect in VWM and in revealing its spatial specificity. However, it leaves open an important question: What enables items seen on trial $n-1$ to interfere on performance in trial $n$ 's VWM? Is it because items on trial $n-1$ were actively maintained in VWM on that trial, or is it because they are simply perceived? Would passively viewed visual information yield as much proactive interference on subsequent VWM tasks? To address these questions, in a followup experiment we revised trial $n-1$ by presenting an array of colors for observers to passively view. After viewing this array for $1,000 \mathrm{msec}$, trial $n$ was presented, including

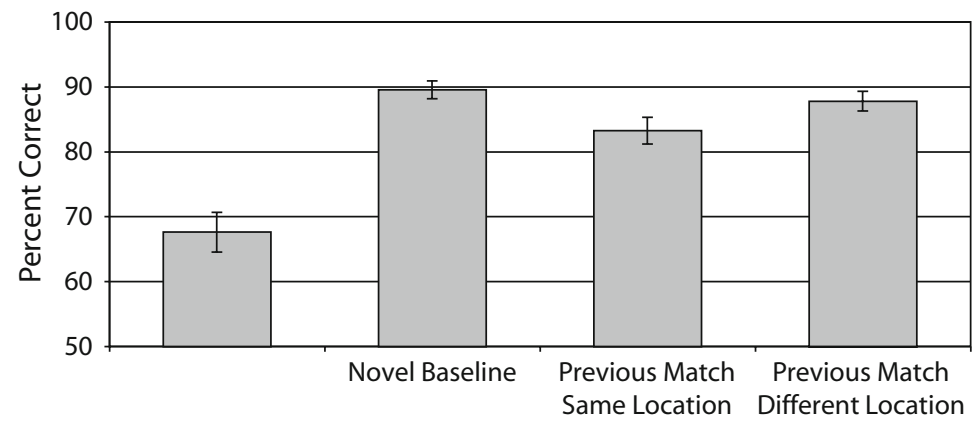

Match

Mismatch

Figure 2. Mean accuracy rates (and $S E$ ) obtained in the memory and passive viewing conditions of Experiment 1, as a function of the type of the test item. 
the same match and mismatch conditions as those used in Experiment 1. Results from that experiment showed no proactive interference from an item passively viewed on trial $n-1$, whether or not the item matched trial $n$ 's probe in location. These results provide the first hint that active memorization of trial $n-1$ 's items may be critical for producing proactive interference on trial $n$. Nonetheless, the follow-up experiment differed from Experiment 1 in trial sequence and task demands, so the difference in results is open to alternative interpretations. Experiment 2 is designed to specifically address whether active memorizing of items on trial $n-1$ is critical for producing proactive interference on subsequent VWM tasks.

\section{EXPERIMENT 2 Visual Proactive Interference in Shape VWM}

Experiment 2 aims to provide converging evidence for the results obtained in Experiment 1. We tested VWM for unnameable novel shapes to verify that proactive interference is not restricted to nameable color stimuli. Experiment 2 also achieves two additional goals. First, to test whether active memory on trial $n-1$ is needed to produce proactive interference on trial $n$, we used a within-subjects design where the only difference between active memory and passive viewing conditions is the task demand. To achieve this goal, we presented observers with six shapes on each trial, but we cued participants' attention to three of the shapes. In Experiment 2A, participants were required to commit the three attended shapes into VWM; in Experiment 2B, participants were required to attend to the three shapes in a demanding visual search task, but they were not required to commit these shapes in VWM. Using this design, we could then probe proactive interference from the attended or the unattended shapes on trial $n-1$.

A second goal of Experiment 2 was to further examine the spatial specificity of proactive interference. In particular, we ask whether proactive interference is tied to the screen location of the previously memorized item or whether it generalizes to a different but configurally equivalent screen location. To address this question, we maintained the spatial configurations of elements from trial $n-1$ to trial $n$ but changed the screen locations by expanding or contracting the configuration. As a result, the probe item on trial $n$ never matched memory items on trial $n-1$ in terms of screen locations. If proactive interference is restricted to the same screen location, we may expect interference to be largely eliminated. However, if proactive interference is configuration based, we should still observe the interference.

\section{Experiment 2A}

\section{Remembering Versus Passive Viewing}

In Experiment 2A, we compared proactive interference from items previously remembered in VWM versus items previously viewed but not attended. Because unattended visual stimuli can result in long lasting priming effects (DeSchepper \& Treisman, 1996), they can potentially result in proactive interference. If VWM on trial $n$ is susceptible to interference from any kind of prior processing, proactive interference should be observed both for items passively viewed and for items remembered on trial $n-1$.

\section{Method}

Participants. Sixteen participants (mean age 23 years) completed Experiment 2A.

Materials. Visual stimuli were drawn from a set of 10 unnameable novel shapes (Shuman \& Kanwisher, 2004) that subtended about $1.3^{\circ} \times 1.3^{\circ}$. On each trial, after a fixation period of $500 \mathrm{msec}$ with a fixation cross $\left(0.41^{\circ}\right)$ and a blank interval of $300 \mathrm{msec}$, an attentional cue display with three white squares (each $1.35^{\circ}$ ) was presented at three randomly selected locations for $200 \mathrm{msec}$. Participants were told to attend to the cued locations and remember the shapes presented there subsequently. After a 400-msec blank interval, the memory array of six shapes was presented for $1,000 \mathrm{msec}$. The shapes were placed equidistantly on an imaginary circle; three of these coincided with the precued locations. The eccentricity of the shapes was the same for all six shapes on a given trial, but the exact value was either $4.92^{\circ}$ or $6.56^{\circ}$, alternating from one trial to another. The alternation resulted in nonoverlapping screen locations from trial $n-1$ 's items to trial $n$ 's, but the configuration was maintained. After a blank retention interval of $1,000 \mathrm{msec}$, a test shape was presented at one of the memorized locations of that trial. The test shape never matched the previous trial's item locations owing to the change of screen locations from adjacent trials.

There were four types of test shapes: match (40\% of trials) and three types of mismatches (60\% of trials). The mismatches were divided randomly and evenly into three conditions: (1) novel baseline, where the test shape was not presented on trial $n$ or trial $n-1$; (2) attended previous match, where the test shape was one of the shapes remembered on trial $n-1$ at the same configural position; and (3) ignored previous match, where the test shape was one of the shapes ignored on trial $n-1$ at the same configural position. Figure 3 illustrates the trial sequence for two adjacent trials. Participants pressed one of two keys to report whether the test item matched the memory item at the probed location on that trial, or whether it was a mismatch. They completed 250 trials of randomly intermixed match and mismatch conditions. The same articulatory suppression procedure as that used in Experiment 1 was employed to reduce verbal recoding.

\section{Results}

Figure 4 shows mean accuracy for different types of probes. Again we focused on mismatch trials, since they were informative of potential proactive interference.

We found proactive interference from trial $n-1$ on VWM of trial $n$, but only for items actively remembered on trial $n-1$. Accuracy was lower in the attended previous match condition than in the novel baseline condition $[t(15)=2.77$, $p<.02]$ or the ignored previous match condition $[t(15)=$ $2.77, p<.02]$. Proactive interference from previously ignored information was statistically insignificant $[t(15)<1]$ for ignored previous match compared with novel baseline.

\section{Discussion}

Experiment 2A confirmed that visual proactive interference was a robust effect that applied not only to color VWM but also to shape VWM. In addition, although proactive interference was spatially specific (Experiment 1), it was not tied to the same screen location, since a probe item that matched trial $n-1$ 's memory item in configural location also led to significant interference. This finding is consistent with the observation that short-term visual memory is configuration based (Carlson-Radvansky \& Irwin, 1995; Jiang, Olson, \& Chun, 2000). Finally, this experiment also showed that merely viewing an item on trial $n-1$ does not 

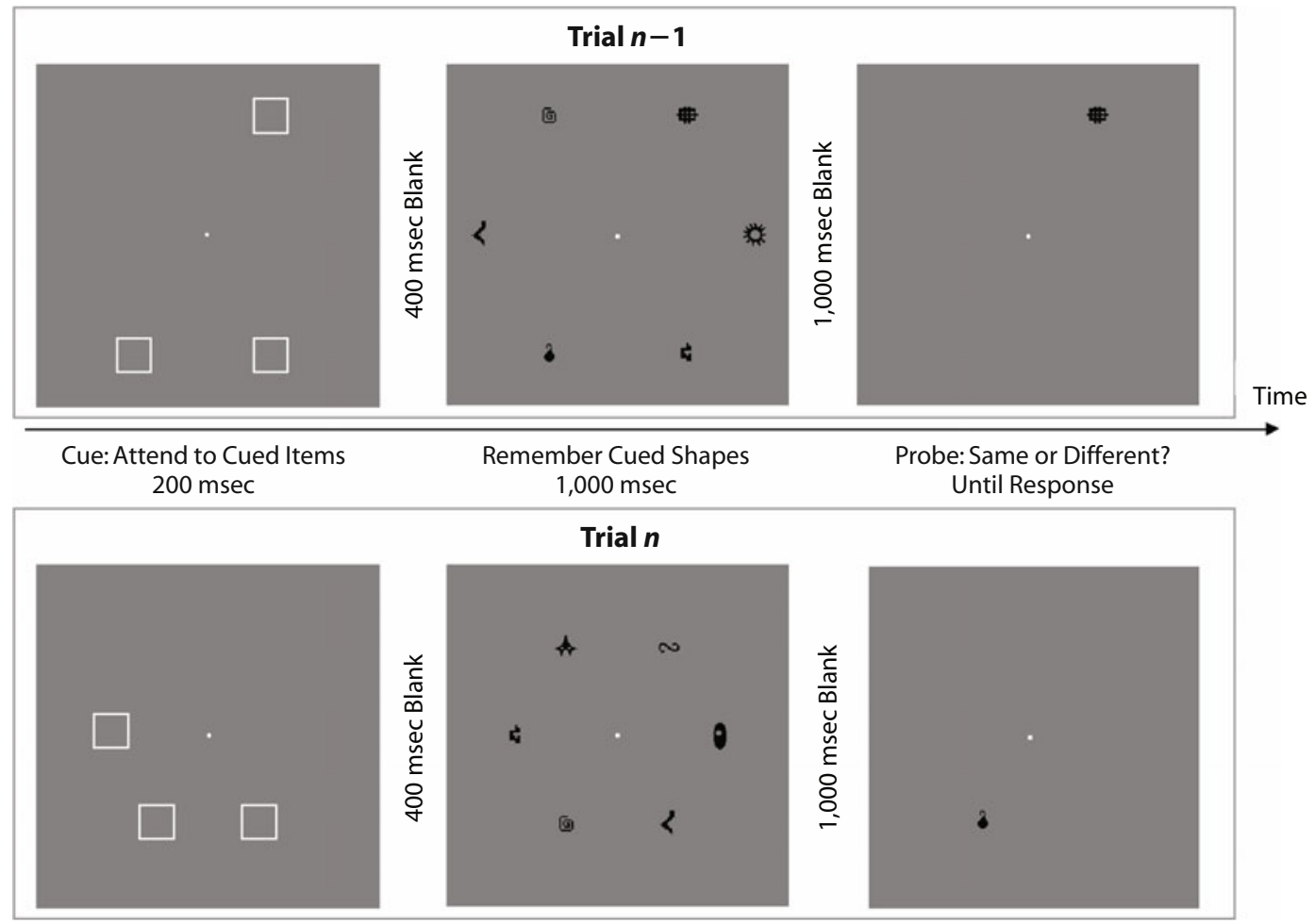

Figure 3. A schematic illustration of trial sequences tested in Experiment 2A. Note that the probe on trial $n$ matched one of the attended items on trial $n-1$ in configural position and shape.

result in proactive interference. Active processing of the items by attending and remembering them in VWM is essential for proactive interference. These results converge onto previous studies in showing that passive viewing and active VWM processing have divergent effects on subsequent visual processing (Makovski, Shim, \& Jiang, 2006; Soto, Heinke, Humphreys, \& Blanco, 2005).

\section{Experiment 2B Attending Without Storing}

Experiment 2A left open the possibility that attending without storing items in VWM could also lead to proac- tive interference. Given the close link between attention and working memory (Woodman \& Luck, 2004), it is important to test whether attending to something is sufficient to produce proactive interference. To achieve this goal, in Experiment $2 \mathrm{~B}$ we required participants to attend to displays on trial $n-1$ without committing these items to VWM. Participants completed a visual search task and a VWM task alternately on consecutive trials. In a similar manner to Experiment $2 \mathrm{~A}$, only half of the items were cued for attention on trial $n-1$ and for memory on trial $n$. The displays and the timing of these tasks were identical to those used in Experiment $2 \mathrm{~A}$. This design allowed us to assess whether attended

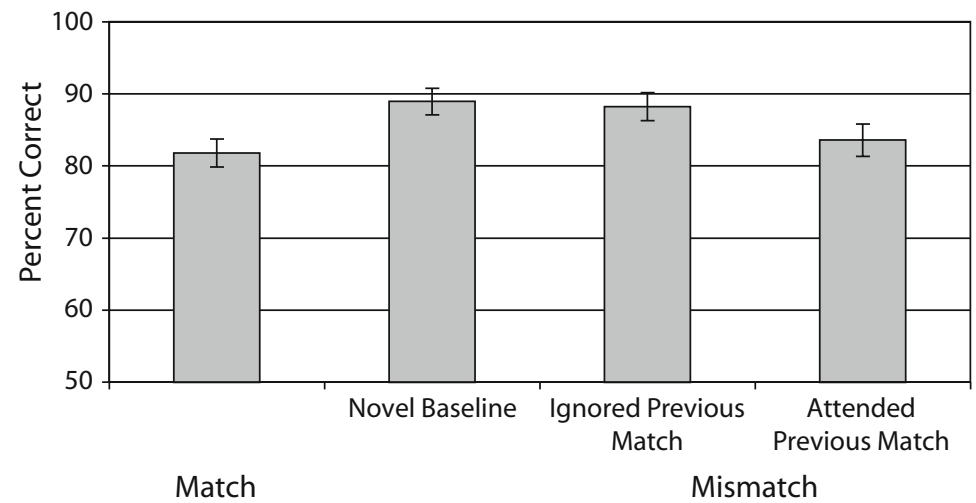

Figure 4. Experiment 2A results: Mean accuracy (and $S E$ ) for different types of test probes. 
but not stored information also yields greater proactive interference than passively viewed information.

\section{Method}

Participants. Thirteen participants (mean age 21 years) completed Experiment 2B.

Materials. The stimuli and procedure used in this experiment were similar to those used in Experiment 2A, including the fact that only three of the six items were task relevant, and that item eccentricity alternated for consecutive trials.

Design. Each participant completed 500 trials, where visual search trials and VWM trials were presented in alternating sequences. Each trial started with a fixation cross together with the task instruction ("remember" for VWM trials and "match" for visual search trials) for $500 \mathrm{msec}$, followed by a 300 -msec blank interval. The VWM task was the same as the one used in Experiment 2A. In the visual search task, participants were instructed to search from the three cued shapes and find the one that matched a central target shape. Here, each trial started with a cue display of three white squares presented at randomly selected locations for $600 \mathrm{msec}$. Then the search display, on which a central target shape and six peripheral shapes were shown, was presented. The three white squares remained on the screen to minimize the demand for remembering the relevant locations. Participants were asked to search from the three cued shapes the one that matched the central target item. The search display was presented for $1,000 \mathrm{msec}$, followed by a probe (in the form of a red disk $1.3^{\circ}$ in diameter) at either the location of the matched item (50\% of trials) or the location of another cued item. Participants were given 1,500 msec to press the " $S$ " key if the probe was at the matched item and the "D" key otherwise. Thus, the visual search trial was comparable with VWM task in timing. The critical difference was that participants were not required to remember the attended items on trial $n-1$ in the search task. Items presented on the visual search task could later appear as the probe on trial $n$, in a similar design as that used in Experiment 2A. Figure 5 illustrates the trial sequence used in Experiment 2B.

\section{Results}

Accuracy in the visual search task was high (mean $=$ 94.4\%), reflecting the fact that visual search was not constrained by the capacity of VWM. Accuracy in the VWM task for different types of probes is plotted in Figure 6.

Unlike in Experiment 2A, in the present experiment there was no evidence for proactive interference from items previously attended on trial $n-1$. Accuracy was not significantly different among the three different mismatch conditions (all $p$ values $>.90$ ).

Direct comparison across Experiments $2 \mathrm{~A}$ and 2B, using experiment as a between-subjects factor and mismatch conditions as a within-subjects factor, revealed a significant interaction between the nature of processing on trial $n-1$ (VWM vs. visual search) and the type of probes (novel baseline vs. attended previous match) $[F(1,27)=$ $4.43, p<.05]$. This finding confirms that active memory is critical for producing proactive interference.

\section{Discussion}

Taken together, Experiments $2 \mathrm{~A}$ and $2 \mathrm{~B}$ provide strong evidence that for information to intrude into subsequent VWM tasks, it needs to be actively remembered previously. Passive viewing and even attending without storing are much less potent at producing proactive interference. However, because attention is strongly related to VWM (Woodman \& Luck, 2004), paying attention to something can sometimes result in incidental memory of that information, which may then be effective at producing proactive interference. Thus, we suggest that active memory is important for producing proactive interference, although this memory may result from either intentional or incidental processing.

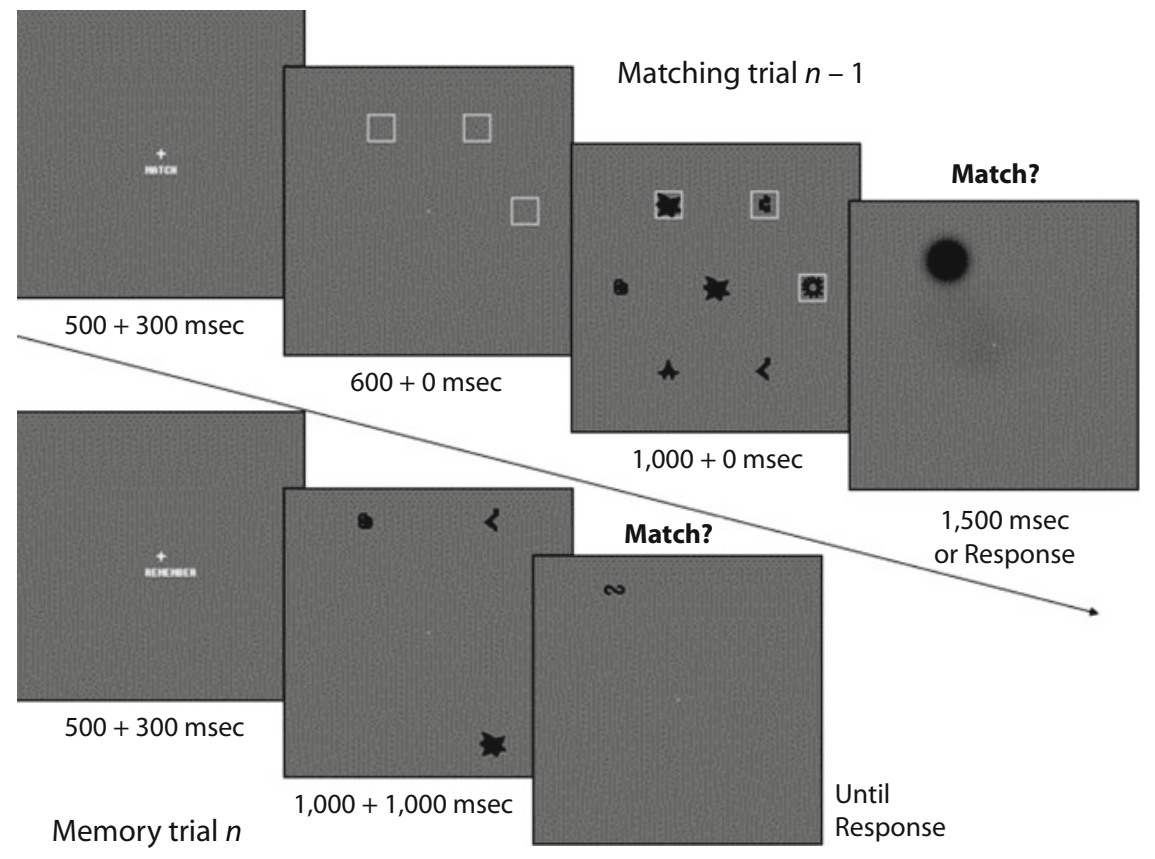

Figure 5. A schematic illustration of trial sequences used in Experiment 2B. 


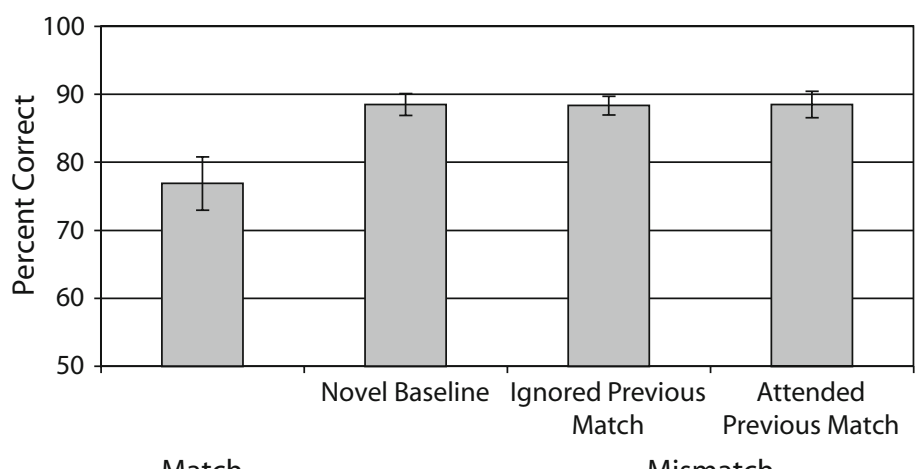

Figure 6. Experiment 2B results: Mean accuracy (and $S E$ ) for different types of test probes.

\section{EXPERIMENT 3 Spatial and Temporal Interference}

So far we have shown that performance on VWM tasks is susceptible to source memory confusions, where memory from trial $n-1$ is confused with memory from trial $n$. The main goal of Experiment 3 is to show that VWM is also susceptible to another source of memory confusion: memory for spatially separate information. Although VWM is configurally organized, with each object tied to a location (Carlson-Radvansky \& Irwin, 1995; Jiang et al., 2000), memory for the binding of object and location can be imprecise, resulting in confusion of memory for adjacent objects (Irwin, 1992; Irwin \& Gordon, 1998; Saiki, 2003). In Experiment 3, we used a four-alternative forced-choice (4AFC) task to probe source memory confusion in VWM. On each trial participants viewed an array of colors. After a short retention interval, a test cue was presented, pointing toward one of the memory locations. Observers had to compare the memory of the cued item with four possible test objects and select the one that matched their memory. The four possible test objects included the actual match, a novel mismatch baseline that differed from colors shown on trial $n-1$ and trial $n$, a mismatch that was the same as trial $n-1$ 's color at the cued location (temporal interference), and a mismatch that was the same as trial $n$ 's color at a location adjacent to the cued item (spatial interfer- ence). Figure 7 shows a schematic illustration of the four types of probes. This experiment examines the strength of source memory interference from spatially or temporally mismatched information on current VWM.

\section{Method}

Participants. Eleven participants (mean age 23 years) completed Experiment 3.

Stimuli and Procedure. We revised the change detection task to a 4AFC task. On each trial, a memory display of six colored disks was presented for $1,000 \mathrm{msec}$. After a blank retention interval of $1,000 \mathrm{msec}$, a test cue in the form of a white arrow $\left(1.44^{\circ}\right.$ in length) was presented at central fixation for $100 \mathrm{msec}$. The arrow pointed at the location of the item that should be retrieved. After a blank interval of $400 \mathrm{msec}$, four test probes were presented in a horizontal array centered at fixation. The center-to-center distance between adjacent probe items was $3.28^{\circ}$. Participants were asked to choose the one that matched their memory at the cued location. The four probes were: (1) the target color (match); (2) the color of a memory item on trial $n$ that was immediately adjacent to the target (spatial interference); (3) the color of a memory item on trial $n-1$ at the location of the cued item (temporal interference); and (4) a novel item not shown on trial $n-1$ or trial $n$ (novel baseline).

Participants completed 280 trials with short breaks between every block of 50 trials. The first trial of a block was excluded from analyses. The procedure and stimuli were otherwise similar to those used in Experiment 1.

\section{Results}

Data from one participant was excluded due to chance performance. Participants picked the actual match on $73 \%$
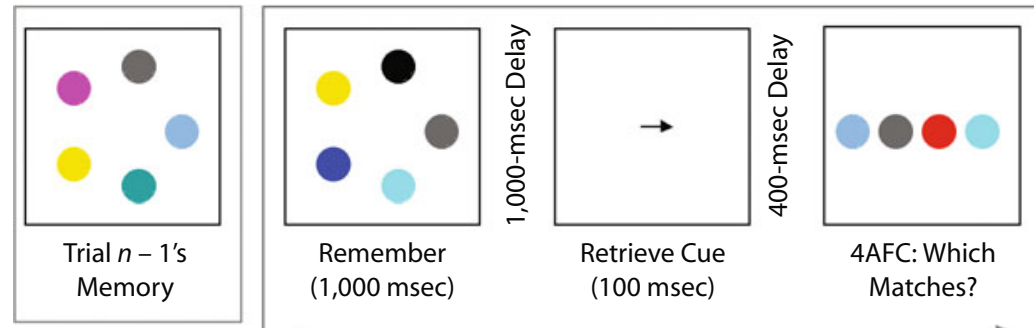

Trial $n$

Figure 7. A schematic illustration of trial sequences used in Experiment 3. The four probe items are (from left to right on this illustration): temporal interference, match, novel baseline, and spatial interference. 
of trials, which was significant above chance level $(p<.01)$. The errors were distributed unevenly across the three types of mismatches, shown in Figure 8. Participants were least likely to misidentify a test item that did not match trial $n-1$ or trial $n$ 's item, the novel baseline. Their false alarm rate significantly increased over the baseline if the probe matched trial $n-1$ 's item at that location, revealing temporal interference $[t(9)=3.02, p<.05]$, or if it matched trial $n$ 's item at the location adjacent to the actual target, showing spatial interference $[t(9)=2.87, p<.05]$. Intrusion from trial $n-1$ 's item at the target location was comparable to intrusion from trial $n$ 's item at an adjacent location $(t<1) .^{2}$

\section{Discussion}

Experiment 3 extended previous studies on VWM by showing that VWM is spatially and temporally imprecise. Spatial imprecision has been shown in past studies in using transsaccadic memory (Irwin, 1992; Irwin \& Andrews, 1996; Irwin \& Gordon, 1998) or change detection paradigms (Saiki, 2003). For example, Irwin (1992) showed that when transsaccadic memory was probed with a partial probe cue, participants tended to commit more within-array errors than extra-array errors. This kind of spatial imprecision is likely due to limited resolution of attention to adjacent elements (Bahcall \& Kowler, 1999). What is unique about our study is that we studied temporal imprecision from preceding trials, whereas most other research on short-term visual memory has only studied effects of temporal delay in the current trial. Temporal delay had little influence on performance in Irwin and other studies (Irwin, 1991; Luck \& Vogel, 1997; Phillips, 1974), suggesting that short-term visual memory does not rapidly decay. Our study has focused on a specific type of interference-proactive interference from the previous trial — and has revealed an aspect of short-term memory not reflected in Irwin's and other visual short-term memory studies. Experiment 3 showed that temporal imprecision is comparable in strength to spatial imprecision in VWM.

\section{GENERAL DISCUSSION}

VWM is considered a window into cognitive control, since successful maintenance and retrieval of information online depends significantly on central executive processes (Baddeley, 2003; Makovski et al., 2006; Vogel, McCollough, \& Machizawa, 2005). Because VWM is limited in capacity (Luck \& Vogel, 1997) and is quickly updated with new visual input (Landman et al., 2003; Makovski \& Jiang, 2007), it would be advantageous to maintain in VWM only currently relevant information. In this study we show that performance in a VWM task is susceptible to proactive interference from items previously held in VWM. The strength of the interference is comparable to that from distractors spatially adjacent to the target. These results highlight the need to consider intertrial effects in studies of VWM.

Previous studies have also explored proactive interference in working memory tasks (for a review, see Jonides $\&$ Nee, 2006), but they are usually tested within the context of an $n$-back task, where participants are required to hold in VWM information presented on previous trials.

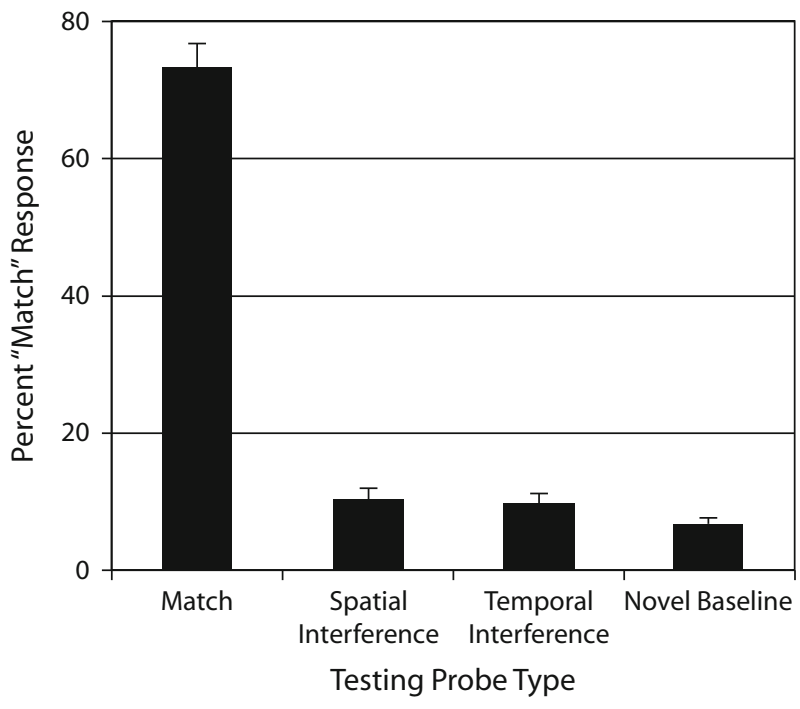

Figure 8. Results from Experiment 3: Frequency distribution (and $S E$ ) for selecting the four types of testing probes as matching trial $n$ memory.

Those that used the recent probe task usually employed verbal or pictorial materials without concurrent articulatory suppression. In the present study, items from previous trials are always irrelevant, so there is no incentive to keep information in VWM beyond the current trial. Even so, proactive interference is observed. This finding reveals two properties of VWM. First, items previously stored in VWM are maintained in memory for longer than required by task demands. Second, in VWM tasks, observers do not have perfect source memory about the time at which memory is acquired. Memory from previous trials is sometimes confused with memory from the current trial, reflecting imperfect control over the content of VWM.

It is important to note that although VWM is not immune to proactive interference, performance is supported primarily by memory from the current trial (see Experiment 3 ). This finding is consistent with observations of visual recency effects, where the most recent visual display dominates the contents of VWM (Broadbent \& Broadbent, 1981; Brockmole, Wang, \& Irwin, 2002; Jiang $\&$ Kumar, 2004). Differences in memory strength for current and previous trials' memory may explain why Olivers et al. (2006) found an effect of the current trial's - but not the previous trial's-VWM on attentional allocation.

What is the source of visual proactive interference? Is the previous trial's memory still actively maintained in VWM, or is it kept elsewhere? There is some hint that the source may be from VWM itself, since proactive interference is negligible from previously attended but not remembered or previously viewed information. Attended visual information is known to result in visual long-term memory (Rock \& Gutman, 1981). Even ignored visual information can lead to long-lasting priming (DeSchepper $\&$ Treisman, 1996). Yet without active working memory processing, information held in other forms of memory seems ineffective in inducing proactive interference. 
Although one is inclined to conclude that proactive interference results from active maintenance of information in VWM after it is no longer needed, there is no strong evidence for this possibility. Information held previously in VWM can continue to occupy VWM, or it may be released from VWM and only retained in visual long-term memory. On the current trial, the previous information may be brought into VWM again, or it may remain outside of VWM. Even in the latter case, where discarded information is not currently in VWM, it can still influence current VWM performance by increasing a sense of familiarity. Just as tasks designed to tap into explicit memory can be achieved by implicit memory (Jacoby, 1991), tasks designed to tap into VWM can also be achieved by other types of memory that enhance a sense of familiarity. To the degree that observers cannot perfectly separate active memory from an enhanced sense of familiarity, VWM performance is open to intrusion from other types of memory. It is empirically difficult to separate contributions to VWM performance from active memory and visual long-term memory. Even a correct response on a given trial may rest on a sense of familiarity. Whether one can borrow Jacoby's procedure of process dissociation (Jacoby, 1991) to separate VWM contribution from visual long-term memory contribution is an important question for future research. The dissociation between VWM contribution and visual long-term memory contribution will not be easy to establish, given that VWM shares many properties with visual long-term memory (Hollingworth, 2005; Olson, Moore, Stark, \& Chatterjee, 2006; Ranganath \& Blumenfeld, 2005). Regardless of exactly where the previous trial's memory is maintained, it is clear that its influence cannot be fully distinguished from the current trial's memory. This memory is also spatially specific, so it is not entirely abstracted from the visual input.

In summary, our study has clearly shown that performance on a VWM task can be influenced not only by the current trial's memory, but also by the previous trial's memory. We propose that humans have limited control over the access of information to VWM.

\section{AUTHOR NOTE}

This research was supported by NSF Grant 0345525 . We thank James Brockmole, Laura Carlson, Tim Vickery, and two anonymous reviewers for comments. Correspondence concerning this article should be directed to T. Makovski, Department of Psychology, University of Minnesota, N218 Elliott Hall, 75 East River Road, Minneapolis, MN 55455 (e-mail: tal.makovski@gmail.com).

\section{REFERENCES}

BADDELEy, A. (2003). Working memory: Looking back and looking forward. Nature Reviews Neuroscience, 4, 829-839.

BAHCALl, D. O., \& Kowler, E. (1999). Attentional interference at small spatial separations. Vision Research, 39, 71-86.

Brainard, D. H. (1997). The Psychophysics Toolbox. Spatial Vision, 10, 433-436.

Broadbent, D. E., \& Broadbent, M. H. (1981). Recency effects in visual memory. Quarterly Journal of Experimental Psychology, 33A, $1-15$.

Brockmole, J. R., Wang, R. F., \& Irwin, D. E. (2002). Temporal integration between visual images and visual percepts. Journal of
Experimental Psychology: Human Perception \& Performance, 28, 315-334.

Carlson-Radvansky, L. A., \& Irwin, D. E. (1995). Memory for structural information across eye movements. Journal of Experimental Psychology: Learning, Memory, \& Cognition, 21, 1441-1458.

Cowan, N. (2001). The magical number 4 in short-term memory: A reconsideration of mental storage capacity. Behavioral \& Brain Sciences, 24, 87-185.

Crowder, R. G. (1976). Principles of learning and memory. Oxford: Erlbaum.

DeSchepper, B., \& Treisman, A. (1996). Visual memory for novel shapes: Implicit coding without attention. Journal of Experimental Psychology: Learning, Memory, \& Cognition, 22, 27-47.

HollingworTh, A. (2005). The relationship between online visual representation of a scene and long-term scene memory. Journal of Experimental Psychology: Learning, Memory, \& Cognition, 31, 396-411.

IRWIN, D. E. (1991). Information integration across saccadic eye movements. Cognitive Psychology, 23, 420-456.

IRWIN, D. E. (1992). Memory for position and identity across eye movements. Journal of Experimental Psychology: Learning, Memory, \& Cognition, 18, 307-317.

IRwIN, D. E., \& ANDREws, R. V. (1996). Integration and accumulation of information across saccadic eye movements. In T. Inui \& J. L. McClelland (Eds.), Attention and performance XVI: Information integration in perception and communication (pp. 125-155). Cambridge, MA: MIT Press.

Irwin, D. E., \& Gordon, R. D. (1998). Eye movements, attention and trans-saccadic memory. Visual Cognition, 5, 127-155.

JАCOBY, L. L. (1991). A process dissociation framework: Separating automatic from intentional uses of memory. Journal of Memory \& Language, 30, 513-541.

Jacoby, L. L., Kelley, C., Brown, J., \& Jasechko, J. (1989). Becoming famous overnight: Limits on the ability to avoid unconscious influences of the past. Journal of Personality \& Social Psychology, 56, 326-338.

JiAnG, Y. H., \& Kumar, A. (2004). Visual short-term memory for two sequential arrays: One integrated representation or two separate representations? Psychonomic Bulletin \& Review, 11, 495-500.

JiAng, Y. H., Olson, I. R., \& Chun, M. M. (2000). Organization of visual short-term memory. Journal of Experimental Psychology: Learning, Memory, \& Cognition, 26, 683-702.

Johnson, M. K. (1997). Source monitoring and memory distortion. Philosophical Transactions of the Royal Society of London: Series B, 352, 1733-1745.

JonidES, J., \& NeE, D. E. (2006). Brain mechanisms of proactive interference in working memory. Neuroscience, 139, 181-193.

Keppel, G., \& Underwood, B. J. (1962). Proactive inhibition in shortterm retention of single items. Journal of Verbal Learning \& Verbal Behavior, 1, 153-161.

Landman, R., Spekreisse, H., \& Lamme, V. A. F. (2003). Large capacity storage of integrated objects before change blindness. Vision Research, 43, 149-164.

LuCK, S. J., \& Vogel, E. K. (1997). The capacity of visual working memory for features and conjunctions. Nature, 390, 279-281.

MAKOVSKI, T., \& JIANG, Y. V. (2007). Distributing versus focusing attention in visual short-term memory. Psychonomic Bulletin \& Review, 14, 1072-1078.

Makovski, T., Shim, W. M., \& Jiang, Y. H. V. (2006). Interference from filled delays on visual change detection. Journal of Vision, $\mathbf{6}$, $1459-1470$.

Monsell, S. (1978). Recency, immediate recognition memory, and reaction time. Cognitive Psychology, 10, 465-501.

Olivers, C. N. L., MeiJer, F., \& Theeuwes, J. (2006). Feature-based memory-driven attentional capture: Visual working memory content affects visual attention. Journal of Experimental Psychology: Human Perception \& Performance, 32, 1243-1265.

Olson, I. R., Moore, K. S., Stark, M., \& Chatterjee, A. (2006). Visual working memory is impaired when the medial temporal lobe is damaged. Journal of Cognitive Neuroscience, 18, 1087-1097.

Pashler, H. (1988). Familiarity and visual change detection. Perception \& Psychophysics, 44, 369-378. 
Pelli, D. G. (1997). The VideoToolbox software for visual psychophysics: Transforming numbers into movies. Spatial Vision, 10, 437-442.

Phillips, W. A. (1974). On the distinction between sensory storage and short-term visual memory. Perception \& Psychophysics, 16, 283-290.

Ranganath, C., \& Blumenfeld, R. S. (2005). Doubts about double dissociations between short- and long-term memory. Trends in Cognitive Sciences, 9, 374-380.

Rensink, R. A. (2002). Change detection. Annual Review of Psychology, 53, 245-277.

Rock, I., \& Gutman, D. (1981). The effect of inattention on form perception. Journal of Experimental Psychology: Human Perception \& Performance, 7, 275-285.

SAIKI, J. (2003). Feature binding in object-file representations of multiple moving items. Journal of Vision, 3, 6-21.

Shuman, M., \& Kanwisher, N. (2004). Numerical magnitude in the human parietal lobe: Tests of representational generality and domain specificity. Neuron, 44, 557-569.

Soto, D., Heinke, D., Humphreys, G. W., \& Blanco, M. J. (2005). Early, involuntary top-down guidance of attention from working memory. Journal of Experimental Psychology: Human Perception \& Performance, 31, 248-261.

Vogel, E. K., McCollough, A. W., \& Machizawa, M. G. (2005). Neural measures reveal individual differences in controlling access to working memory. Nature, 438, 500-503.
Woodman, G. F., \& Luck, S. J. (2004). Visual search is slowed when visuospatial working memory is occupied. Psychonomic Bulletin \& Review, 11, 269-274.

\section{NOTES}

1. Because all conditions were randomly intermixed in testing sequence, we could not divide the source of errors on match trials; that is, when participants mistook a match trial as a mismatch, we could not know whether they mistook it for a novel item or for a previous memory. For this reason, we cannot calculate $d^{\prime}, a^{\prime}$, or $\beta$, since the hit rate is not independently assessed for different conditions; nor can we calculate memory capacity using Pashler's or Cowan's method for capacity estimation.

2. These results were replicated in a subsequent experiment that included one more mismatch condition - an item presented on trial $n-1$ at a location spatially adjacent to trial $n$ 's target. That experiment revealed a similar pattern of results in the spatial interference and temporal interference conditions, although intrusion was weakened to an insignificant level when the probe was displaced in both space and time.

(Manuscript received March 10, 2007; revision accepted for publication July 16, 2007.) 